\title{
Dasatinib is safe and effective in unselected chronic myeloid leukaemia elderly patients resistant/intolerant to imatinib
}

\author{
Roberto Latagliata $^{\mathrm{a}, *}$, Massimo Breccia ${ }^{\mathrm{a}}$, Fausto Castagnetti ${ }^{\mathrm{b}}$, Fabio Stagno $^{\mathrm{c}}$, Luigiana Luciano $^{\mathrm{d}}$, \\ Antonella Gozzini ${ }^{\mathrm{e}}$, Stefano Ulisciani ${ }^{\mathrm{f}}$, Francesco Cavazzini ${ }^{\mathrm{g}}$, Mario Annunziata ${ }^{\mathrm{h}}$, Federica Sorà ${ }^{\mathrm{i}}$, \\ Antonella Russo Rossi ${ }^{\mathrm{j}}$, Patrizia Pregno ${ }^{\mathrm{k}}$, Enrico Montefusco ${ }^{1}$, Elisabetta Abruzzese ${ }^{\mathrm{m}}$, Elena Crisà ${ }^{\mathrm{n}}$, \\ Pellegrino Musto $^{\circ}$, Mario Tiribelli ${ }^{p}$, Gianni Binotto ${ }^{\mathrm{q}}$, Ubaldo Occhini ${ }^{\mathrm{r}}$, Costanzo Feo ${ }^{\mathrm{s}}$, Paolo Vigneri ${ }^{\mathrm{c}}$, \\ Valeria Santini $^{\mathrm{e}}$, Carmen Fava ${ }^{\mathrm{f}}$, Giannantonio Rosti ${ }^{\mathrm{b}}$, Giuliana Alimena ${ }^{\mathrm{a}}$
}

a Dipartimento di Biotecnologie Cellulari ed Ematologia, Università “La Sapienza”, Via Benevento 6, 00161, Rome, Italy

${ }^{\mathrm{b}}$ Ematologia, Università di Bologna, Bologna, Italy

${ }^{\mathrm{c}}$ Ematologia, Ospedale Ferrarotto, Catania, Italy

d Ematologia, Università "Federico II", Napoli, Italy

e Ematologia, Università di Firenze, Firenza, Italy

f Ematologia, Polo Universitario ASO San Luigi Gonzaga, Orbassano, Italy

g Ematologia, Università di Ferrara, Ferrara, Italy

h Ematologia, Ospedale Cardarelli, Napoli, Italy

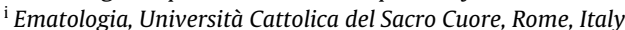

${ }^{\mathrm{j}}$ Ematologia con Trapianto, Università di Bari, Bari, Italy

k Ematologia, Ospedale S Giovanni Battista, Torino, Italy

${ }^{1}$ Ematologia, Ospedale Sant'Andrea, Rome, Italy

m Ematologia, Ospedale Sant'Eugenio, Rome, Italy

${ }^{\mathrm{n}}$ Divisione di Ematologia, Università di Torino, Torino, Italy

${ }^{\circ}$ Dipartimento Onco-Ematologico, IRCCS, Centro di Riferimento Oncologico della Basilicata, Rionero in Vulture, Vulture, Italy

${ }^{\mathrm{p}}$ Ematologia, Università di Udine, Udine, Italy

${ }^{\mathrm{q}}$ Ematologia, Università di Padova, Padova, Italy

${ }^{\mathrm{r}}$ Ematologia, Ospedale San Donato, Arezzo, Arezzo, Italy

${ }^{s}$ DH Ematologico, AO Rummo, Benevento, Italy

\section{A R T I C L E I N F O}

\section{Article history:}

Received 3 March 2011

Received in revised form 6 May 2011

Accepted 10 May 2011

Available online 25 June 2011

\section{Keywords:}

Chronic Myeloid Leukaemia

Dasatinib

Elderly

Safety

\begin{abstract}
A B S T R A C T
To highlight dasatinib role in the elderly, 125 unselected patients with CP-CML aged >60 years resistant/intolerant to imatinib were retrospectively evaluated. Grade 3-4 haematological and extrahaematological toxicities were reported in 39 (31.2\%) and 34 (27.2\%) patients; grade 3-4 haematological toxicity was higher in patients with $140 \mathrm{mg}$ starting dose $(50.0 \%$ vs $19.6 \%, p=0.001)$. Grade $3-4$ pleuropericardial effusions occurred in 10 patients $(8.0 \%)$. Dose reductions were more common in patients with $140 \mathrm{mg}$ ( $88.4 \%$ vs $26.7 \%, p<0.001)$. Of 122 evaluable patients, $72(59.1 \%)$ had cytogenetic response [12 (9.8\%) partial, 60 (49.3\%) complete]. Overall, 38/60 patients in complete CyR also achieved a molecular response. Cumulative OS at 24 and 48 months were 93.1\% (95\% CI 88.4-97.8) and 84.2\% (95\% CI 74.6-93.7). Dasatinib, at the recommended dose of $100 \mathrm{mg} / \mathrm{day}$, is effective and safe also in unselected elderly subjects.
\end{abstract}

(C) 2011 Elsevier Ltd. All rights reserved.

\section{Introduction}

Imatinib, the prototype tyrosine kinase inhibitor (TKI) versus the $\mathrm{BCR} / \mathrm{ABL}$ hybrid gene, has profoundly changed the prognosis of Chronic Myeloid Leukaemia (CML) and is the current standard first-line treatment for patients with newly diagnosed CML [1-3].

\footnotetext{
* Corresponding author. Tel.: +39 06 857954537; fax: +39 0644241984.

E-mail address: rob.lati@libero.it (R. Latagliata).
}

However, some patients discontinue the drug due to treatment failure or toxicity [4,5]. As a consequence, there is increasing effort focused on overcoming both resistance and intolerance to imatinib.

Dasatinib is a 2nd generation TKI with greater potency than imatinib on BCR/ABL tyrosine-kinase [6,7]. Many phase II trials with dasatinib in patients resistant or intolerant to imatinib have confirmed its efficacy with an acceptable safety profile [8-10]; furthermore, the dosage of $100 \mathrm{mg}$ once daily emerged in a 4-arm randomized trial as the best tolerated dosage for standard treatment in chronic phase patients [11]. 
There are few studies which focused on the role of imatinib in older adults and elderly patients [12-14]. In the MDACC report [12], comparable rates of complete cytogenetic response (CCyR) were observed in older (>60 yrs) versus younger patients; on the contrary, lower rates of response (hematologic and cytogenetic) in older ( $>65 \mathrm{yrs}$ ) versus younger patients were observed in the GIMEMA study [14]. However in both studies the survival rate was similar.

Nevertheless, very limited data are available in older adults and elderly patients resistant/intolerant to imatinib and treated with a 2nd generation TKI. In addition, only selected elderly patients have been enrolled in phase II published trials, with a median age lower than expected according to epidemiological studies and a rate of patients aged $>60$ years often $<35-40 \%$ [15].

Elderly people in onco-haematological settings are extremely heterogenous. If we look at controlled clinical trials, we can get information on treatment tolerability and efficacy in fit elderly patients who are compliant with treatments, not in the whole elderly population; therefore, to have a "real-life" evaluation with useful informations for current clinical practice, we need to gather data from the entire group of elderly patients.

The aim of our study was to describe the tolerability and the efficacy of dasatinib in a wide unselected cohort of older adult patients with CML resistant or intolerant to imatinib collected by several haematological Centers in Italy.

\section{Patient and methods}

2.1. Inclusion criteria

Participating Centers were asked to send data on all CML patients resistant/intolerant to Imatinib receiving dasatinib who fulfilled at the start of dasatinib treatment the following inclusion criteria, irrespectively of their enrolment or not in controlled clinical trials;

\author{
- Age $>60$ yrs \\ - Chronic phase of disease
}

Intolerance to imatinib was defined as the occurrence of severe (grade 3-4 WHO) or moderate (grade $2 \mathrm{WHO}$ ) but persistent or recurrent haematological or extra-haematological toxicity during imatinib treatment requiring permanent drug discontinuation. Primary resistance to imatinib was defined according to European LeukaemiaNet guidelines for failure at 3 months [no complete haematological response (CHR)], 6 months (no cytogenetic response), 12 months [less than partial cytogenetic response (PCyR)] and 18 months (no CCyR) [16]. Secondary resistance to imatinib was defined as the occurrence of loss of CCyR or loss of major molecular response (MMolR) at any time after the achievement of a previous CCyR or MMolR.

Any clinical illness requiring a specific and prolonged treatment as well as previous cancers was considered as concomitant disease. The number of drugs required by each patient to manage concomitant diseases was also recorded.

Haematological and extra-haematological toxicities were graded according to the WHO scale; for the purpose of the present study, only severe toxicities (grade 3-4) were considered.

\subsection{Cytogenetic and molecular evaluation}

Cytogenetic analyses were performed on bone marrow (BM) aspirates by standard $\mathrm{G}$ or $\mathrm{Q}$ banding techniques on at least 20 cell metaphases from direct or short term $(24-48 \mathrm{~h})$ cultures at diagnosis, after 3,6 , and 12 months of therapy with dasatinib and every 6 months thereafter. Fluorescence - in situ hybridization (FISH) on bone marrow interphase cells was recommended if less than 20 metaphases were evaluable and was performed with BCR-ABL extra-signal, dual-color, dual-fusion probes; CCgR was defined by less than 2 of $200(<1 \%)$ positive marrow cell interphase nuclei.

Real-time quantitative polymerase chain reaction (RT-Q-PCR) to assess BCR-ABL transcript levels was performed according to suggested procedures and recommendations and results were expressed as BCR-ABL/ABL ratio normalised according to international scale (IS) [17-19].

Qualitative RT-nested PCR was carried out according to the standardized RT-PCR analysis of fusion gene transcripts as previously described in the BIOMED-16 report.
Table 1

Clinical characteristics at CML diagnosis.

\begin{tabular}{ll}
\hline No. of patients & 125 \\
Males, No. (\%) & $63(50.6 \%)$ \\
Age (years): median (interquartile range) & $63.1(58.6-69.2)$ \\
WBC(x 10 $/ 1):$ median (interquartile range) & $63.1(30.1-117.0)$ \\
Hb (g/dl): median (interquartile range) & $12.2(10.5-13.3)$ \\
PLTS (x 109/l): median (interquartile range) & $396(277-608)$ \\
Sokal risk: No. & \\
Low & 32 \\
Intermediate & 49 \\
High & 19 \\
Not evaluable & 25 \\
\hline
\end{tabular}

\subsection{Definitions}

Cytogenetic and molecular responses were categorized according to standard criteria: CCyR was defined as the absence of Ph+ metaphases; if less than 20 metaphases were evaluable, CCyR was defined as BCR-ABL positive interphase nuclei less than 1\%; partial cytogenetic response (PCyR) was defined as the presence of 1 to $35 \% \mathrm{Ph}+$ metaphases; MMolR was defined as $\mathrm{BCR}-\mathrm{ABL} / \mathrm{ABL}$ ratio $<0.1$ and complete molecular response (CMolR) as the undetectability of BCR/ABL by qualitative RT-nested PCR.

Primary haematological resistance to dasatinib was defined as failure to achieve CHR after 3 months of treatment; primary cytogenetic resistance to dasatinib was defined as failure to achieve at least PCyR after 12 months of treatment. Secondary resistance to dasatinib was defined as the loss of cytogenetic response at any time after the achievement of a previous PCyR or CCyR.

\subsection{Statistical analysis}

Data were expressed as mean \pm standard deviation (SD) (normally distributed data), median and interquartile range (IR) (non-normally distributed data), or as percentage frequencies, and within-patient comparisons were made by paired test and $\chi^{2}$ test, as appropriate, at significance levels of $p<0.05$. Overall Survival (OS) was calculated from the date of dasatinib start to death due to any cause. EventFree Survival (EFS) was calculated from the date dasatinib was started to any of the following events: primary haematological resistance to dasatinib, permanent dasatinib discontinuation due to toxicity or any other unrelated cause, secondary resistance to dasatinib, death due to any cause. The Cox proportional hazard model was used for multivariate analysis of prognostic factors.

All calculations were made using a standard statistical package (SPSS for Windows Version 15.0; Chicago, IL).

\section{Results}

\subsection{Clinical characteristics}

Overall, 125 patients resistant/intolerant to imatinib and treated with dasatinib when aged $>60$ years were treated by 21 Italian haematological Centers, of which 11 were university hospitals and 10 community-based hospitals. The territorial distribution of the participating Centers was nationwide. Patient clinical characteristics at CML diagnosis are shown in Table 1: as expected in a relatively aged population, intermediate and high Sokal scores were frequent.Median interval from diagnosis to start of dasatinib treatment was 75.8 months (IR 39.1-115.9) while median age at dasatinib initiation was 69.9 years (IR 65.4-74.4). As to previous treatments, 57 patients (45.6\%) had received an interferon-based therapy before imatinib. According to the inclusion criteria, all 125 patients had received imatinib, which was administered at standard daily dosage of $400 \mathrm{mg}$ in 116 patients and at a reduced daily dosage of $<400 \mathrm{mg}$ in 9 patients; in addition, 58 patients $(46.4 \%)$ escalated the dose to $600-800 \mathrm{mg}$. Overall, the median duration of imatinib treatment was 46.6 months (IR 21.8-61.8). Prior to switch to dasatinib, 12 patients $(9.6 \%)$ received treatment with nilotinib and 16 patients $(12.8 \%)$ with other drugs. In particular, among the 12 patients previously treated with nilotinib, 8 discontinued nilotinib for primary resistance, 3 for cytogenetic relapse and 1 for intolerance.

At the start of dasatinib treatment, 13 patients (10.4\%) resulted intolerant for extra-haematological toxicity to imatinib (skin 
Table 2

Concomitant diseases at start of dasatinib treatment.

$\begin{array}{lc}\text { Arterial hypertension } & 54(43.2 \%) \\ \text { Cardiovascular diseases } & 22(17.6 \%) \\ \text { Previous or concomitant neoplasia } & 11(8.8 \%) \\ \text { Gastro-intestinal diseases } & 10(8.0 \%) \\ \text { Arrhytmias } & 9(7.2 \%) \\ \text { Thyroid disfunctions } & 9(7.2 \%) \\ \text { Diabetes } & 9(7.2 \%) \\ \text { BPCO } & 7(5.6 \%) \\ \text { Chronic renal insufficiency } & 4(3.2 \%)\end{array}$

toxicity in 5, gastro-intestinal toxicity in 3, asthenia in 2 and liver toxicity, pleural effusion and arterial stenosis in 1 case, respectively) and 112 (89.6\%) resistant to imatinib (primary resistance in 60 and secondary resistance in 52 patients, respectively). On the whole, 11 patients were in CCyR when dasatinib was started; of them, 6 were intolerant to imatinib, 4 had a secondary resistance due to loss of MMolR and 1 had a loss of MMolR with F359V mutation. According to ECOG scale, performance status was 0 in 67 patients (53.6\%), 1 in 48 (38.4\%) and $\geq 2$ in 10 (8.0\%), respectively.

A major point in defining our cohort of patients was the presence of concomitant diseases. Twenty-seven patients (21.6\%) did not have any concomitant disease, 48 patients (38.4\%) had 1 concomitant disease and 50 patients $(40.0 \%)$ had 2 or more concomitant diseases when dasatinib was started. Table 2 shows the concomitant diseases most frequently reported, possibly interacting with TKI intake, metabolism and side-effects.

Strictly related to the concomitant diseases, we also report the number of concomitant drugs taken by patients at the start of dasatinib treatment. Only 29 patients (23.2\%) did not take any concomitant drug, 57 patients $(45.6 \%)$ received $1-3$ concomitant drugs while 39 patients $(31.2 \%$ ) needed 4 or more different drugs.

Starting daily dose of Dasatinib was $140 \mathrm{mg}$ in 52 patients, $100 \mathrm{mg}$ in 56 patients and less than $100 \mathrm{mg}$ in the remaining 17 patients. Drug intake was once daily in 79 patients (63.2\%) and twice daily in 46 (36.8\%), respectively.

\subsection{Treatment toxicities}

After a median period of treatment of 20.7 months (range 0.7-61.5; IR 10.4-29.2) all patients were evaluable for toxicity. Overall, 39/125 patients (31.2\%) developed a severe haematological toxicity (grade 3-4 according to WHO) after a median treatment period of 1.2 months (IR 0.7-2.4).

Regarding the starting dosage, a grade 3-4 haematological toxicity was reported in $26 / 52$ patients $(50.0 \%$ ) treated with a daily dose of $140 \mathrm{mg}$ compared to $11 / 56$ (19.6\%) patients treated with $100 \mathrm{mg}$ $(p=0.001)$. Differences were also significant for thrombocytopenia and neutropenia, as shown in Table 3. Furthermore, at univariate analysis also female gender $(p=0.01)$, primary resistance $(p=0.018)$ and twice daily administration $(p=0.002)$ had a bad prognostic impact on grade 3-4 haematological toxicity. At multivariate analysis, only starting dosage retained statistical significance $(p=0.014)$.

Severe extra-haematological toxicity (grade 3-4 according to WHO) was reported in $34 / 125$ patients (27.2\%) after a median treatment period of 2.3 months (IR 0.9-6.9). According to starting dosage, $16 / 52$ (30.7\%) patients treated with $140 \mathrm{mg}$ developed a severe extra-haematological toxicity compared to $12 / 56(21.4 \%)$

Table 3

Haematological toxicities according to starting dose.

\begin{tabular}{lclc}
\hline & 140 mg No. $(\%)$ & $100 \mathrm{mg}$ No. $(\%)$ & $p$-Value \\
\hline $\mathrm{Hb}<8$ g/dl & $9(17.3)$ & $4(7.1)$ & 0.10 \\
$\mathrm{PLTS}<50 \times 109 / 1$ & $17(32.6)$ & $3(5.3)$ & $<0.001$ \\
$\mathrm{PMN}<1.0 \times 109 / 1$ & $15(28.8)$ & $4(7.1)$ & 0.003 \\
\hline
\end{tabular}

Table 4

Extra-haematological toxicities according to starting dose.

\begin{tabular}{llll}
\hline Toxicity 3-4 WHO & $140 \mathrm{mg}$ No. (\%) & $100 \mathrm{mg}$ No. (\%) & $p$-Value \\
\hline Pleural effusion & $4(7.7)$ & $5(8.9)$ & $\mathrm{NS}$ \\
Bone pain & $2(3.8)$ & 0 & $\mathrm{NS}$ \\
DVT & $2(3.8)$ & 0 & $\mathrm{NS}$ \\
Miocardial ischemia & $1(1.9)$ & 0 & $\mathrm{NS}$ \\
Bronchopneumonia & $1(1.9)$ & $2(3.5)$ & $\mathrm{NS}$ \\
Cheratocongiuntivitis & $1(1.9)$ & 0 & $\mathrm{NS}$ \\
G.I. hemorrhage & $1(1.9)$ & 0 & $\mathrm{NS}$ \\
Skin rush & $1(1.9)$ & 0 & $\mathrm{NS}$ \\
Ascytis & $1(1.9)$ & 0 & $\mathrm{NS}$ \\
Sepsis & 0 & $1(1.7)$ & $\mathrm{NS}$ \\
Diarrhea & 0 & $1(1.7)$ & $\mathrm{NS}$ \\
Abdominal pain & 0 & $1(1.7)$ & $\mathrm{NS}$ \\
Neurological symptoms & 0 & $1(1.7)$ & NS \\
Dysgeusia & 0 & $1(1.7)$ & NS \\
Fluid retention & 0 & 0 & NS \\
\hline
\end{tabular}

patients treated with $100 \mathrm{mg}(p=0.26)$. No other feature at baseline showed a prognostic impact on extra-haematological toxicity.

Pleuro-pericardial effusions of any WHO grade occurred in $41 / 125$ patients $(32.8 \%$ ) and were severe (grade $3-4$ ) in $10 / 125$ patients $(8.0 \%)$; with regard to initial dosage, pleural effusions of any WHO grade developed in 25/52 (48.0\%) patients treated with $140 \mathrm{mg}$ compared to $13 / 56$ (23.2\%) patients treated with $100 \mathrm{mg}(p=0.01)$. The incidence of severe (grade 3-4 WHO) pleuro-pericardial effusion and other extra-haematological toxicities related to initial dose of dasatinib is summarized in Table 4.

The number of concomitant diseases as well as the number of concomitant drugs did not affect significantly the incidence of grade 3-4 haematological and extra-haematological toxicity (Supplemental Table 1). Furthermore, it is worth of note that among patients starting dasatinib due to imatinib intolerance, no case of cross-intolerance was reported.

During treatment, 58 patients (46.4\%) did not require any dose adjustment and 67 patients (53.6\%) needed a dose reduction due to toxicity; 19 patients (15.2\%) required permanent dasatinib discontinuation due to toxicity, after a median period from dasatinib start of 4.0 months (IR 2.2-6.0). However, a different profile of dose modification was observed according to the initial dose of dasatinib, with $46 / 52$ patients ( $88.4 \%$ ) requiring dose reduction in the group receiving $140 \mathrm{mg}$ compared with $15 / 56$ patients (26.7\%) receiving $100 \mathrm{mg}(p<0.001)$

\subsection{Treatment response and follow-up}

With regard to response, 122 patients were considered evaluable ( $\geq 3$ months of treatment) and 3 were considered as too early. In terms of best response, these were as follows: 16 patients $(13.1 \%)$ did not achieve any response (including 12 patients with early dasatinib discontinuation for toxicity and 1 patient who died from unrelated 2nd neoplasia); 34 patients (27.8\%) had a CHR only, but a cytogenetic response less than partial; 72 patients (59.1\%) had a cytogenetic response (12 PCyR, 60 CCyR). Among 60 patients with CCyR, 38 (31.1\% of all 122 evaluable patients) also achieved Molecular Response (19 MMolR, 19 CMolR). Results according to starting dosage are shown in Table 5.

At univariate analysis, baseline features with a poor prognostic impact on CCyR achievement were primary resistant disease $(p<0.001)$, twice daily drug administration $(p=0.004)$ and bad PS $(p=0.024)$ : there was also a trend for $140 \mathrm{mg}$ initial dose as bad prognostic factor $(p=0.052)$. On the contrary, the number of concomitant diseases and the number of concomitant drugs (Supplemental Table 2), gender, Sokal risk and previous IFN treatment did not affect significantly cytogenetic and molecular 
Table 5

Best response according to starting dose.

\begin{tabular}{lccc}
\hline & All pts & $140 \mathrm{mg}$ & $100 \mathrm{mg}$ \\
\hline Evaluable patients & 122 & 52 & 55 \\
No chr & $16(13.1 \%)$ & $7(13.4 \%)$ & $3(5.5 \%)$ \\
Chr only & $34(27.8 \%)$ & $18(34.6 \%)$ & $13(23.6 \%)$ \\
Cytogenetic response & $72(59.1 \%)$ & $27(52.0 \%)$ & $39(70.9 \%)$ \\
PCyR & 12 & 5 & 7 \\
CCyR & $60(49.1 \%)$ & $22(42.3 \%)$ & $32(58.1 \%)$ \\
Molecular response & $38(31.1 \%)$ & $14(26.9 \%)$ & $20(36.3 \%)$ \\
MMolR & 19 & 6 & 11 \\
CMolR & 19 & 8 & 9 \\
\hline
\end{tabular}

response rates. At multivariate analysis, only baseline disease status $(p<0.001)$ and PS $(p=0.014)$ retained statistical significance.

After a median period from start of Dasatinib treatment of 24.4 months (IR 17.3-32.9), 11 patients died from disease progression (2) or other causes (from cardiac disease 3 patients, from infection 2 patients, from 2 nd neoplasia 2 patients, from acute renal failure 1 patient and from unknown cause 1 patient) and 1 patient was lost to follow-up. The remaining 113 patients are still alive.

The cumulative OS at 24 and 48 months were $93.1 \%$ (95\% CI 88.4-97.8) and 84.2\% (95\% CI 74.6-93.7), respectively (Fig. 1a), without differences according to starting dosage (Fig. 1b). The only feature affecting OS at univariate analysis was PS at baseline $(p=0.0001)$.

The cumulative EFS at 24 and 48 months were $60.8 \%(95 \% \mathrm{CI}$ 51.2-70.4) and $49.5 \%$ (95\% CI 37.3-61.7), respectively; according to starting dosage, there was no difference between patients receiving 140 or $100 \mathrm{mg}$, while both these groups had a better EFS compared to patients receiving $<100 \mathrm{mg}(p=0.032$ ) (Fig. $2 \mathrm{a}$ and b). At univariate analysis, other baseline features with a poor prognostic impact on EFS were primary resistant disease $(p=0.0023)$, and bad PS $(p=0.021)$ : there was also a trend for twice daily administration as bad prognostic factor ( $p=0.059$ ). On the contrary, gender, Sokal risk and previous IFN treatment did not affect significantly EFS.
At multivariate analysis, starting dosage $(p<0.001)$ and baseline disease status $(p=0.006)$ retained statistical significance.

\section{Discussion}

Treatment results with 2nd generation TKI in patients resistant/intolerant to Imatinib has already been reported in many phase II-III trials over the past 2 years [8-11]. However, data specifically addressing results and toxicities in elderly patients are still lacking and, therefore, the primary aim of this study was to give new insights in the 2nd line treatment of these subjects with dasatinib.

We described here a "real-life" cohort of patients, instead of a cohort from a phase II-III controlled trial, inviting participant Centers to collect data on all elderly patients treated at each institution with dasatinib, irrespectively of the enrolment or not in a controlled trial. Patients enrolled in such controlled trials are selected according to many inclusion and exclusion criteria. This selective bias is evident especially among older patients, as reported by Rohrbacher [15], and confirmed by the median age in these trials, which is often $<55$ years. In our cohort, 10 patients $(8.0 \%)$ had a baseline poor performance status, 12 patients (9.6\%) were previously treated with a 2nd generation TKI inhibitor, 22 patients (17.6\%) had cardiological concomitant diseases and 4 patients (3.2\%) had chronic renal insufficiency; many of these patients would have been excluded from a phase II-III controlled trial. It is virtually impossible to obtain a cohort without any form of selection, as some patients considered too frail by responsible physicians are probably excluded from second-line treatment with TKI; therefore, a collection of clinical data outside controlled trials may be considered a "real-life evaluation" and could be the best method to investigate the feasibilty of second-line TKI in this subset of patients.

Moving to this "real-life" source, we can discuss our results trying to answer three questions; (1) is dasatinib safe in elderly people in common clinical practice? (2) which is the best dose of dasatinib in this setting? (3) are the therapeutic results sufficient to justify dasatinib treatment also in this setting?
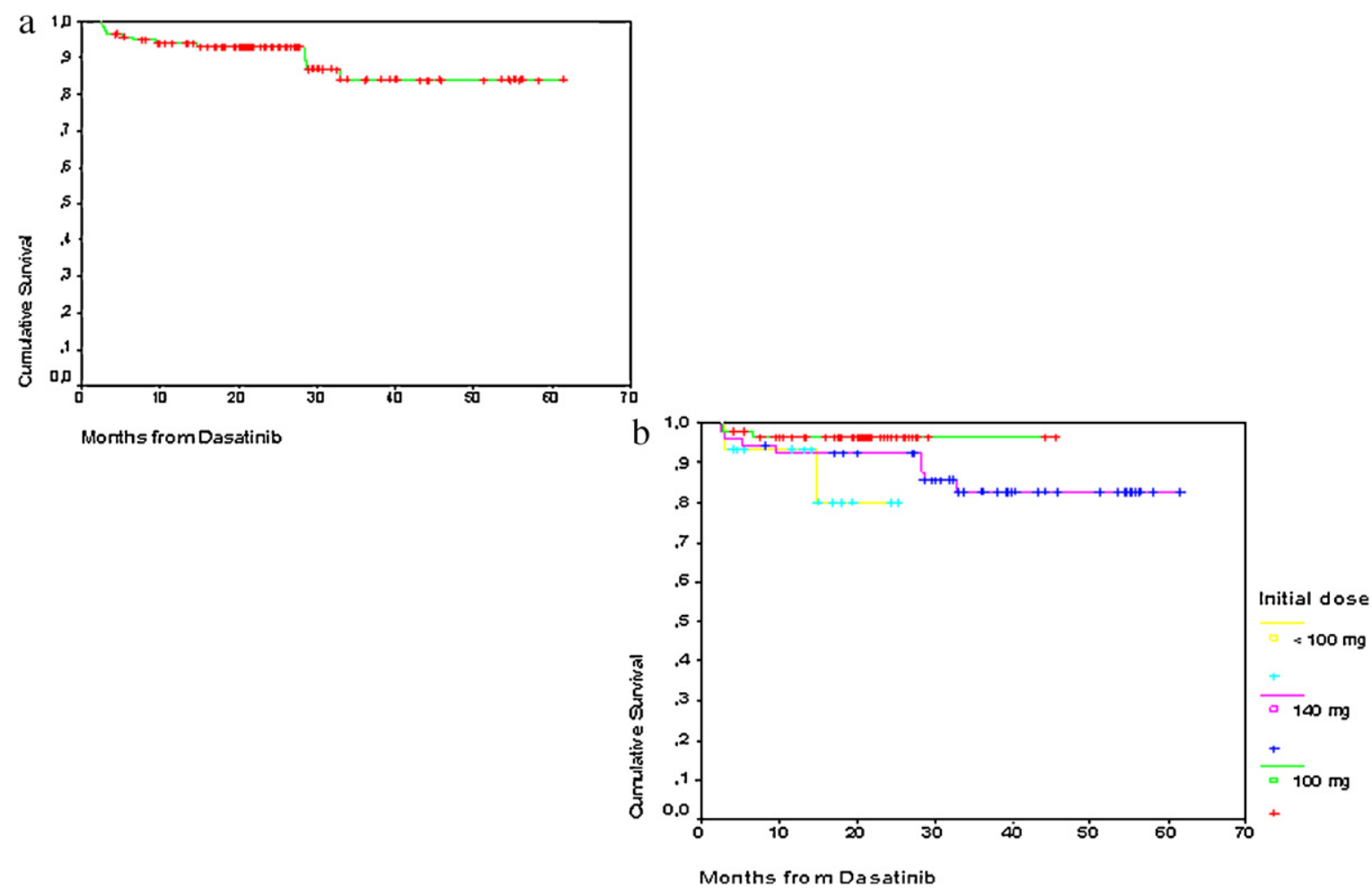

Fig. 1. (a) Cumulative overall survival (b) cumulative overall survival according to starting dose. 

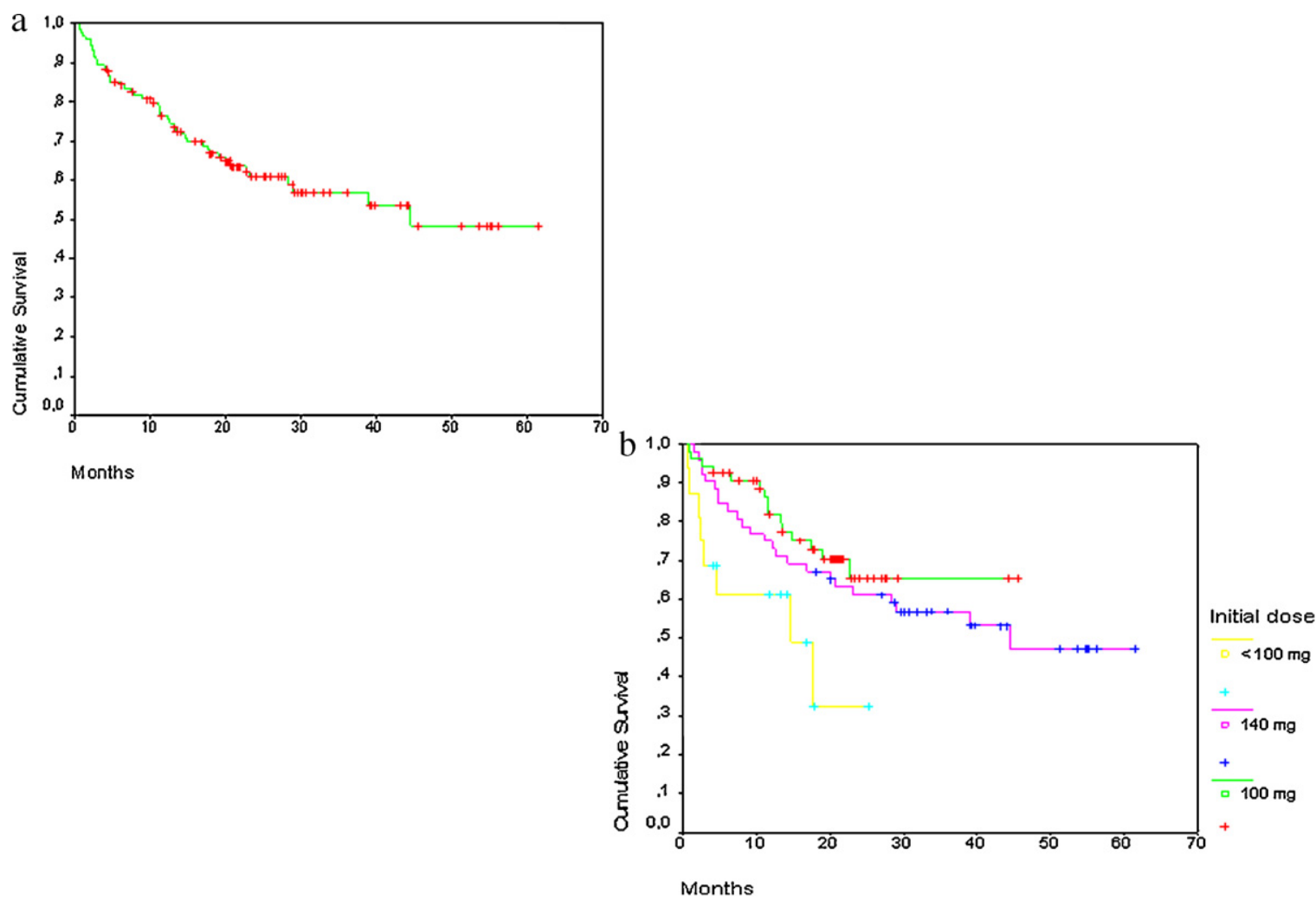

Fig. 2. (a) Cumulative event-free survival (b) cumulative overall event-free survival according to starting dose.

Overall, both haematological and extra-haematological grade 3-4 toxicities as well as the rate of permanent discontinuation due to toxicity (15.2\%) were acceptable, considering the high rate of patients with concomitant diseases and receiving concomitant drugs. It is worth of note that both safety and efficacy of Dasatinib were not affected by the number of concomitant diseases and drugs of the patients.

As expected, the initial dose of dasatinib had a crucial role in determining the toxicity and compliance to treatment [11]. The majority of patients starting dasatinib at $140 \mathrm{mg}$ needed to reduce the dosage compared to patients who received lower doses; in addition, the initial dosage of $100 \mathrm{mg}$ was associated with a lower incidence of grade 3-4 neutropenia and thrombocytopenia when compared to $140 \mathrm{mg}$. On the contrary, probably due to the low number of patients, grade 3-4 extra-haematological toxicities were similar according to the initial dose of dasatinib.

The overall incidence of pleural effusions was higher in patients receiving $140 \mathrm{mg}$, in line with data from the 034 trial [11]; however, the difference disappeared when only grade 3-4 pleural effusions were considered.

Response rates were higher than expected considering the unselected cohort and the older age of patients. Overall results were similar to data previously reported in phase II trials [8-11], showing a CCyR rate of about $50 \%$ and a cumulative OS $>80 \%$ at 4 years but, unlike in other trials, in the present analysis secondary resistance was defined either as cytogenetic and molecular relapse. It is worth noting that response rates in patients who received $100 \mathrm{mg}$ as initial dasatinib dosage were higher (although not significantly different), with a CCyR rate of about $60 \%$ and a cumulative OS $>95 \%$ at 4 years; a longer follow-up and a wider cohort of patients are needed to address the possible favourable impact of this dosage on treatment results.

In conclusion, dasatinib seems to be a safe and effective option for elderly patients resistant/intolerant to imatinib, irrespective of any selection criteria and especially at the current standard initial dosage of $100 \mathrm{mg}$.

As a matter of fact, moving from this "real-life" approach, we outline that there is no reason to treat elderly CML patients resistant/intolerant to Imatinib with a conservative approach only, excluding them from a safe and effective drug like dasatinib. Our "real-life" data in elderly are also promising if we point at the future use of 2nd generation TKIs in the 1st line CML treatment.

\section{Conflict of interest statement}

GR is a consultant with Novartis and serves on the speakers' bureaus of Novartis and Bristol-Myers Squibb and all other authors have no disclosure to declare.

\section{Acknowledgements}

Funding source. There was no financial support to declare.

Contributions. RL, MB and GA designed the data collection and wrote the paper; FC, FS, LL, AG, SU, FC, MA, FS, ARR, PP, EM, EA EC, PM, MT, GB, UO, CF collected data and contributed to the data analysis and interpretation and PV, VS, CF, GR analysed data and contributed to write the paper.

\section{Appendix A. Supplementary data}

Supplementary data associated with this article can be found, in the online version, at doi:10.1016/j.leukres.2011.05.015.

\section{References}

[1] Druker BJ, Guilhot F, O’Brien SG, Gathmann I, Kantarjian H, Gattermann N et al. Five-year follow-up of patients receiving imatinib for chronic myeloid leukemia. N Engl J Med 2006;355:2408-17. 
[2] Branford S, Seymour J, Grigg A, Arthur C, Rudzki Z, Lynch K, et al. BCR-ABL messenger RNA levels continue to decline in patients with chronic phase chronic myeloid leukemia treated with imatinib for more than 5 years and approximately half of all first-line treated patients have stable undetectable BCR-ABL using strict sensitivity criteria. Clin Cancer Res 2007;13:7080-6.

[3] Hochhaus A, Druker B, Sawyers C, Guilhot F, Schiffer CA, Cortes J, et al. Favorable long-term follow-up results over six years for response, survival and safety with imatinib mesylate therapy in chronic phase chronic myeloid leukemia post failure of interferon-alpha treatment. Blood 2008;111:1039-43.

[4] Branford S, Rudzki Z, Walsh S, Parkinson I, Grigg A, Szer J, et al. Detection of BCR-ABL mutations in patients with CML treated with imatinib is virtually always accompanied by clinical resistance, and mutations in the ATP phosphate-binding loop (P-loop) are associated with a poor prognosis. Blood 2003;102:276-83.

[5] Kujawski L, Talpaz M. Strategies for overcoming imatinib resistance in chronic myeloid leukemia. Leuk Lymph 2007;48:2310-22.

[6] O'Hare T, Walters DK, Stoffregen EP, Jia T, Manley PW, Mestan J, et al In vitro activity of Bcr-Abl inhibitors AMN107 and BMS-354825 against clinically relevant imatinib-resistant Abl kinase domain mutants. Cancer Res 2005;65:4500-5.

[7] Tokarski JS, Newitt JA, Chang CY, Cheng JD, Wittekind M, Kiefer SE, et al. The structure of dasatinib (BMS-354825) bound to activated ABL kinase domain elucidates its inhibitory activity against imatinib-resistant ABL mutants. Cancer Res 2006;66:5790-7.

[8] Hochhaus A, Kantarjian HM, Baccarani M, Lipton JH, Apperley JF, Druker BJ, et al. Dasatinib induces notable hematologic and cytogenetic responses in chronic phase chronic myeloid leukemia after failure of imatinib therapy. Blood 2007;109:2303-9.

[9] Hochhaus A, Baccarani M, Deininger M, Apperley JF, Lipton JH, Goldberg SL, et al. Dasatinib induces durable cytogenetic responses in patients with chronic myelogenous leukemia in chronic phase with resistance or intolerance to imatinib. Leukemia 2008;22:1200-6.

[10] Kantarjian HM, Pasquini R, Levy V, Jootar S, Holowiecki J, Hamerschlak N, et al. Dasatinib or high-dose imatinib for chronic-phase chronic myeloid leukemia resistant to imatinib at a dose of 400 to 600 milligrams daily: two-year followup of a randomized Phase 2 study (START-R). Cancer 2009;115:4136-47.

[11] Shah NP, Kim DW, Kantarjian HM, Rousselot P, Llacer PE, Enrico A, et al Potent, transient inhibition of BCR-ABL with dasatinib $100 \mathrm{mg}$ daily achieves rapid and durable cytogenetic responses and high transformation-free survival rates in chronic phase chronic myeloid leukemia patients with resistance, suboptimal response or intolerance to imatinib. Haematologica 2010;95: 232-40.

[12] Cortes J, Talpaz M, O’Brien S, Giles F, Beth Rios M, Shan J, et al. Effects of age on prognosis with imatinib mesylate therapy for patients with Philadelphia chromosome-positive chronic myelogenous leukemia. Cancer 2003;15(98:):1105-13.

[13] Latagliata R, Breccia M, Carmosino I, Sarlo C, Montefusco E, Mancini M, et al. Older patients with $\mathrm{Ph}+$ chronic myelogenous leukemia (CML): results of imatinib mesylate treatment. Leuk Res 2005;29:287-91.

[14] Rosti G, Iacobucci I, Bassi S, Castagnetti F, Amabile M, Cilloni D, et al. Impact of age on the outcome of patients with chronic myeloid leukemia in late chronic phase: results of a phase II study of the GIMEMA CML Working Party. Haematologica 2007;92:101-5.

[15] Rohrbacher M, Berger U, Hochhaus A, Metzgeroth G, Adam K, Lahaye T, et al. Clinical trials underestimate the age of chronic myeloid leukemia (CML) patients. Incidence and median age of $\mathrm{Ph} / \mathrm{BCR}-\mathrm{ABL}$-positive $\mathrm{CML}$ and other chronic myeloproliferative disorders in a representative area in Germany. Leukemia 2009;23:602-4.

[16] Baccarani M, Saglio G, Goldman J, Hochhaus A, Simonsson B, Appelbaum F, et al. Evolving concepts in the management of chronic myeloid leukemia. Recommendations from an expert panel on behalf of the European Leukemianet. Blood 2006;108:1809-18.

[17] Hughes T, Deininger M, Hochhaus A, Branford S, Radich J, Kaeda J, et al. patients responding to treatment with tyrosine kinase inhibitors: review and recommendations for harmonizing current methodology for detecting BCRABL transcripts and kinase domain mutations and for expressing results. Blood 2006;108:28-37.

[18] Branford S, Fletcher L, Cross NC, Muller MC, Hochhaus A, Kim DW, et al. Desirable performance characteristics for BCR-ABL measurement on an international reporting scale to allow consistent interpretation of individual patient response and comparison of response rates between clinical trials. Blood 2008;112:3330-8.

[19] Muller MC, Erben P, Saglio G, Gottardi E, Nyvold CG, Schenk T, et al. Harmonization of BCR-ABL mRNA quantification using a uniform multifunctional control plasmid in 37 international laboratories. Leukemia 2008;22:96-102. 